\title{
Acute Transverse Myelitis Following COVID-19 Vaccination
}

\author{
Jhih-Jian Gao ${ }^{1}$, Hung-Pin Tseng ${ }^{1}$, Chun-Liang Lin ${ }^{1}$, Jr-Shiang Shiu ${ }^{2}$, Ming-Hsun Lee ${ }^{3}$ \\ and Ching-Hsiung Liu ${ }^{1, *}$ \\ 1 Department of Neurology, Lotung Poh-Ai Hospital, Ilan 26546, Taiwan; c087001@mail.pohai.org.tw (J.-J.G.); \\ 1913@mail.pohai.org.tw (H.-P.T.); c923001@mail.pohai.org.tw (C.-L.L.) \\ 2 Department of Emergency Medicine, Lotung Poh-Ai Hospital, Ilan 26546, Taiwan; \\ mrblack@mail.pohai.org.tw \\ 3 Department of Radiology, Lotung Poh-Ai Hospital, Ilan 26546, Taiwan; 985003@mail.pohai.org.tw \\ * Correspondence: chl@mail.pohai.org.tw; Tel.: +886-3-954-3131
}

Citation: Gao, J.-J.; Tseng, H.-P.; Lin, C.-L.; Shiu, J.-S.; Lee, M.-H.; Liu, C.-H. Acute Transverse Myelitis Following COVID-19 Vaccination. Vaccines 2021, 9, 1008. https://doi.org/10.3390/ vaccines 9091008

Academic Editor: Giovanni Ferrara

Received: 31 July 2021

Accepted: 7 September 2021

Published: 10 September 2021

Publisher's Note: MDPI stays neutral with regard to jurisdictional claims in published maps and institutional affiliations.

Copyright: (c) 2021 by the authors. Licensee MDPI, Basel, Switzerland. This article is an open access article distributed under the terms and conditions of the Creative Commons Attribution (CC BY) license (https:/ / creativecommons.org/licenses/by/ $4.0 /)$.

\begin{abstract}
An increasing number of people are undergoing vaccination for COVID-19 because of the ongoing pandemic. The newly developed, genetically engineered mRNA vaccines are critical for controlling the epidemic disease. However, major adverse effects, including neuroimmunological disorders, are being attributed to this vaccine. For instance, several cases of acute transverse myelitis (ATM) after COVID-19 vaccination have been reported in clinical trials. Here, we report an exceedingly rare case of longitudinally extensive transverse myelitis (LETM), a rare subtype of ATM involving three or more vertebral segments, that occurred shortly after vaccination with the Moderna COVID-19 (mRNA-1273) vaccine, with a comorbidity of vitamin B12 deficiency. The findings of subsequent investigations suggest the possibility that autoimmune responses are triggered by the reactions between anti-SARS-CoV-2 spike protein antibodies and tissue proteins, as well as the interaction between spike proteins and angiotensin-converting enzyme 2 receptors.
\end{abstract}

Keywords: myelitis; LETM; COVID-19; COVID-19 vaccination; mRNA vaccine; vitamin B12

\section{Introduction}

Acute transverse myelitis (ATM) is a disease characterized by weakness, sensory disturbances, and bladder and bowel dysfunction. A rare subtype of ATM, longitudinally extensive transverse myelitis (LETM), refers to the inflammation of the spinal cord, involving three or more vertebral segments [1,2]. As the disease progresses, it may cause severe disability. Patients with ATM are categorized as falling under parainfectious, multiple sclerosis, spinal cord ischemia, or idiopathic causes [3].

Cases of ATM after vaccination were also reported on post-vaccination days 5-28 [4]. Although ATM is a sporadic neurological disorder associated with the COVID-19 pandemic globally, ATM following COVID-19 vaccination is rare. As the adoption of COVID-19 vaccines became prevalent worldwide, reports of their adverse effects began to emerge. The American Neurological Association (ANA) investigated the major neurological complications of COVID-19 vaccination, including tremors, diplopia, tinnitus, dysphonia, seizures, the reactivation of herpes zoster, transverse myelitis, stroke, Bell's palsy, acute disseminated encephalomyelitis, and Guillain-Barré syndrome [5]. Here, we report an exceedingly rare case of LETM that occurred shortly after vaccination with the Moderna COVID-19 (mRNA-1273) vaccine.

\section{Case Presentation}

A 76-year-old female was admitted to our neurological ward with presentations of unsteadiness and abnormal sensation in the limbs, predominantly on the right side. There were no major significant comorbidities aside from hypertension and right-sided hearing impairment. Six days before admission, she had received COVID-19 vaccine (mRNA-1273, 
Moderna) in the morning (9 a.m.), after which she experienced intermittent low-grade fever (approximately $37-38^{\circ} \mathrm{C}$ ) in the evening $(4 \mathrm{p} . \mathrm{m}$.). Additionally, she was found to have right upper limb paresthesia that extended from the distal to the proximal limb areas, and to the right lower limb on post-vaccination day 2. She had no symptoms of mental deterioration, headache, neck rigidity, vertigo, nausea, vomiting, fecal or urinary disturbance. There was no history of vegetarianism, gastric surgery, or nitrous oxide exposure. However, progressive gait disturbance and sacral paresthesia occurred on post-vaccination day 3 (Figure 1).

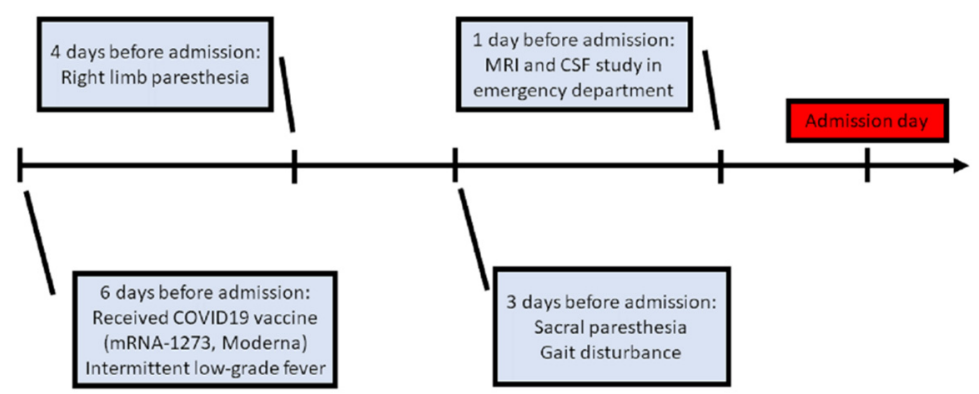

Figure 1. Clinical course before admission. (MRI: magnetic resonance imaging; CSF: cerebrospinal fluid).

On examination, she exhibited relatively good muscle strength, but also decreased proprioceptive sensation below the right T4 dermatome. The patient also exhibited impairment in joint position sense and thermal analgesia in the right limbs. The deep tendon reflex of the right limbs was relatively brisk. The Babinski sign showed a right extensor plantar response.

A neuroimaging study of the brain and cervical cord was performed on post-vaccination day 5, using magnetic resonance imaging (MRI). C-spine MRI revealed extensive intramedullary hyperintensity in the cervical cord at the $\mathrm{C} 2-\mathrm{C} 5$ levels on T2-weighted images, and at the $\mathrm{C} 3$ level with $\mathrm{T} 1$ ring enhancement of the cervical cord (Figure 2). Brain MRI and magnetic resonance angiography showed no abnormal findings. Cerebrospinal fluid (CSF) analysis showed mild pleocytosis $(15 / \mu \mathrm{L})$ with neutrophil predominance $(73 \%)$ and increased protein levels $(57.2 \mathrm{mg} / \mathrm{dL}$; normal limit: $15-45 \mathrm{mg} / \mathrm{dL})$. CSF rapid plasma reagin (RPR), treponema pallidum hemagglutination (TPPA), human immunodeficiency virus (HIV), and cytology were all negative. The multiplex polymerase chain reaction (PCR) assay FilmArray meningitis/encephalitis panel did not detect pathogens such as bacteria, viruses, and fungi in the central nervous system. Rheumatoid factor and antinuclear antibody levels were within normal limits. Serum anti-aquaporin 4 (AQP4) antibodies were negative. Somatosensory evoked potential and nerve conduction studies revealed bilateral peroneal neuropathy. The motor and visual evoked potentials were normal. Brainstem auditory evoked potentials showed right sensorineural hearing impairment. The visual evoked potential study revealed a non-significant finding. The CSF was negative for the oligoclonal band. Additionally, analyses for detecting connective tissue disorders and vasculitis profiles revealed no abnormalities (Table 1).

Based on the Brighton case definition for myelitis, the patient was diagnosed with LETM with level 2 diagnostic certainty [6]. Pulse therapy with intravenous methylprednisolone ( $1 \mathrm{~g} /$ day for five days) was initiated after admission. The patient showed improvement in limb and sacral paresthesia symptoms after the pulse therapy, following which, oral prednisolone $(60 \mathrm{mg} /$ day $)$ was administered. The examinations also revealed a deficiency in vitamin B12 levels, at $131 \mathrm{pg} / \mathrm{mL}$. Therefore, hydroxocobalamin (1 mg/day) was included in the regimen to compensate for the deficiency. Since the patient gradually regained sensation in the limbs affected by paresthesia, prednisolone was slowly tapered. Her gait also improved following rehabilitation. The patient was discharged two weeks after admission with oral prednisolone, $15 \mathrm{mg}$ twice daily. It was tapered to $10 \mathrm{mg}$ twice daily 1 week later during outpatient department treatment. A follow-up cervical MRI 
two weeks after discharge showed that the T2 hyperintensity had decreased in size, with minimal enhancement in the T1-weighted image (Figure 3). Prednisolone was tapered to $5 \mathrm{mg}$ three times daily. It was further tapered to $10 \mathrm{mg}$ once daily, four weeks after discharge. Her gait improved without the assistance of a cane.
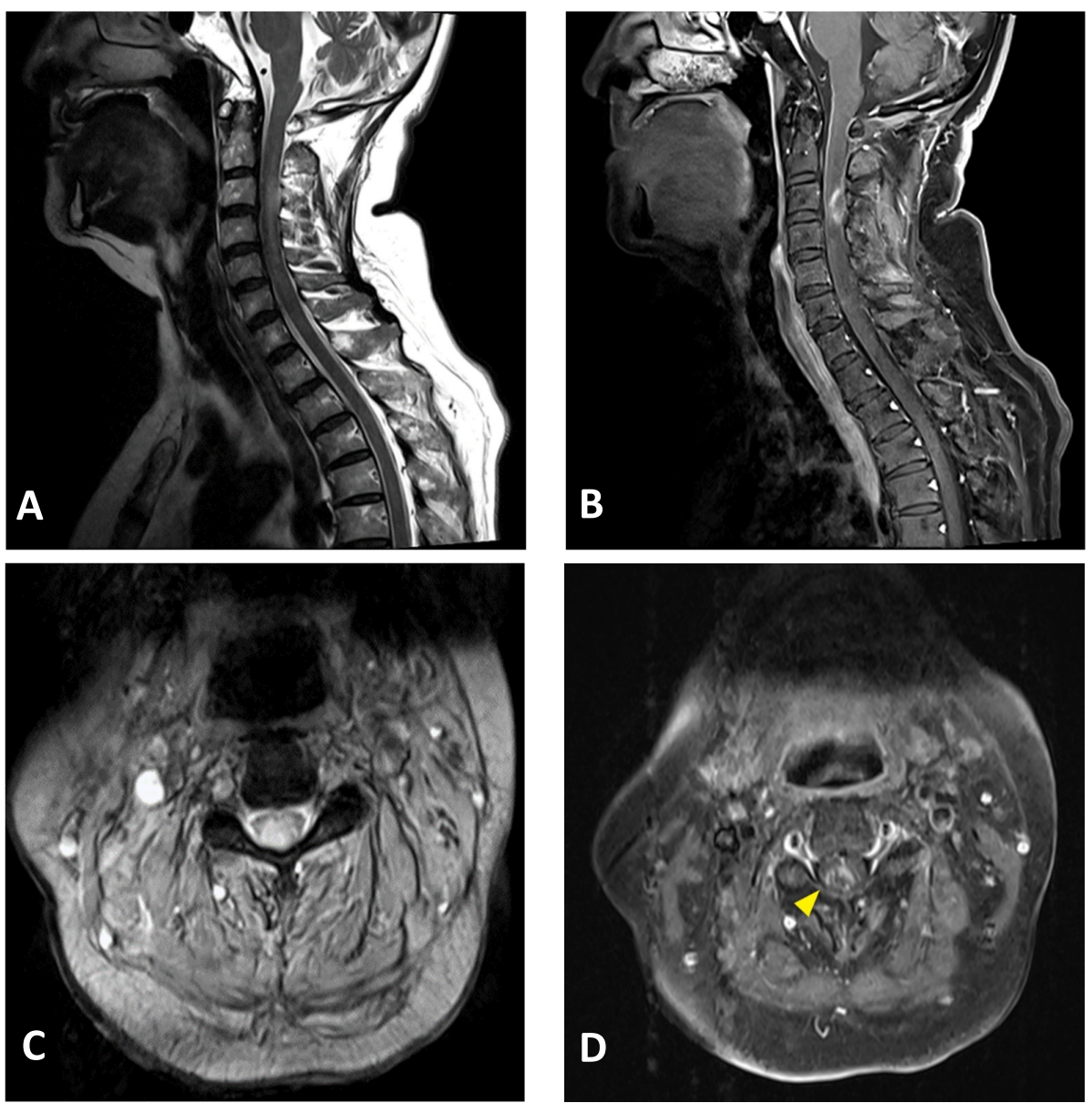

Figure 2. Cervical MRI images of a 76-year-old female with longitudinally extensive transverse myelitis: (A) sagittal T2-weighted image showing hyperintensity in the cervical cord at the C2-C5 levels; (B) sagittal T1-weighted image with contrast showing ring enhancement in the cervical cord at the C3 level; (C) axial T2-weighted image showing extensive hyperintensity in the cervical cord at the C3 level; (D) axial T1-weighted image with contrast showing right-sided enhancement in the cervical cord at the C3 level (arrowhead).

Table 1. Laboratory profile.

\begin{tabular}{ccc}
\hline Parameter [Unit] & Result & Reference Value \\
\hline WBC $\left[10^{9} / \mathrm{L}\right]$ & 4.60 & $4.50-11.00$ \\
Hgb $[\mathrm{g} / \mathrm{dL}]$ & 12.0 & $12.00-16.0$ \\
Platelet $\left[10^{9} / \mathrm{L}\right]$ & 296 & $150-400$ \\
MCV [fL] & 113.7 & $80.0-96.0$ \\
WBC CSF $[/ \mu \mathrm{L}]$ & 15 & \\
RBC CSF $[\mu \mathrm{L}]$ & 73 & $28-96$ \\
Lymphocyte CSF [\%] & 27 & \\
Neutrophil CSF [\%] & 73 & $<2.8$ \\
Lactate CSF $[\mathrm{mmol} / \mathrm{L}]$ & 2.7 & $15.0-45.0$ \\
Protein CSF $[\mathrm{mg} / \mathrm{dL}]$ & 57.2 & $40-70$ \\
Glucose CSF $[\mathrm{mg} / \mathrm{dL}]$ & 71 &
\end{tabular}


Table 1. Cont.

\begin{tabular}{|c|c|c|}
\hline Parameter [Unit] & Result & Reference Value \\
\hline Glucose serum $[\mathrm{mg} / \mathrm{dL}]$ & 117 & \\
\hline ESR $[\mathrm{mm}]$ & 27 & $0-20$ \\
\hline $\mathrm{CRP}[\mathrm{mg} / \mathrm{dL}]$ & 0.06 & $<0.50$ \\
\hline Anti-HIV EIA [COI] & 0.11 & $<0.9$ \\
\hline TPPA CSF & Negative & Negative \\
\hline RPR/VDRL CSF & Non-reactive & Non-reactive \\
\hline Electrophoresis CSF & No oligoclonal band & \\
\hline Vitamin B12 [pg/mL] & 131 & Deficient $<211$ \\
\hline $\mathrm{RF}[\mathrm{IU} / \mathrm{mL}]$ & $<10.0$ & $<14.0$ \\
\hline ANA & $1: 40(-)$ & \\
\hline $\mathrm{TSH}[\mu \mathrm{IU} / \mathrm{mL}]$ & 0.18 & $0.270-4.20$ \\
\hline Free T4 [ng/dL] & 1.57 & $0.93-1.70$ \\
\hline A-beta2GPI IgG [U/mL] & $<1.4$ & $<20.0 \mathrm{U} / \mathrm{mL}$ \\
\hline Anti-cardiolipin IgG [GPL-U/mL] & $<1.6$ & $<20.0$ \\
\hline ENA anti-SSA [AI] & $<0.2$ & $<1.0$ \\
\hline ENA anti-SSA 52 [AI] & $<0.2$ & $<1.0$ \\
\hline ENA anti-SSA 60 [AI] & $<0.2$ & $<1.0$ \\
\hline ENA anti-SSB [AI] & $<0.2$ & $<1.0$ \\
\hline cANCA & $10 \times$ (Negative) & \\
\hline pANCA & $10 \times($ Negative $)$ & \\
\hline Atypical pANCA & $10 \times$ (Negative) & \\
\hline Anti-dsDNA [IU/mL] & $<1.0$ & Negative $<4$ \\
\hline TSH receptor $\mathrm{Ab}[\mathrm{IU} / \mathrm{L}]$ & $<0.10$ & Negative $<0.1$ \\
\hline Aquaporin 4 antibody & Negative & Negative \\
\hline
\end{tabular}

WBC: white blood cells count; Hgb: hemoglobin; MCV: mean corpuscular volume; RBC: red blood cells; CSF: cerebrospinal fluid; ESR: erythrocyte sedimentation rate; CRP: C-reactive protein; HIV: human immunodeficiency virus; EIA: enzyme-linked immunoassay; TPPA: treponema pallidum particle agglutination; RPR: rapid plasma regain; VDRL: venereal disease research laboratory; RF: rheumatoid factor; ANA: antinuclear antibody; TSH: thyroid stimulating hormone; T4: thyroxine; GPI: glycoprotein I; IgG: immunoglobin G; ENA: extractable nuclear antigen; SSA: Sjögren's syndrome-related antigen A; SSB: Sjögren's syndrome-related antigen B; cANCA: cytoplasmic anti-neutrophil cytoplasmic autoantibodies; pANCA: perinuclear anti-neutrophil cytoplasmic autoantibodies; dsDNA: double stranded deoxyribonucleic acid.
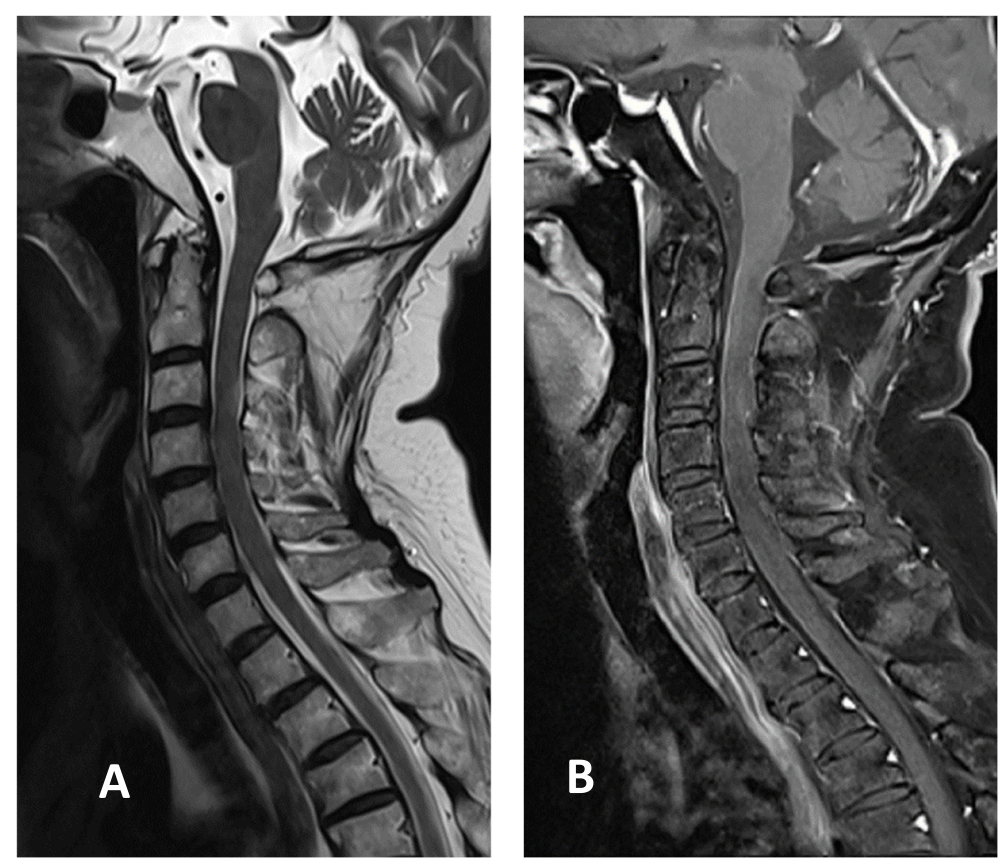

Figure 3. Cervical MRI images two weeks after discharge: (A) sagittal T2-weighted image showing the decreased size of hyperintensity in the cervical cord at the C3 level; (B) sagittal T1-weighted image with contrast showing minimal enhancement in the cervical cord at the C3 level. 


\section{Discussion}

We present the first case of myelitis (LETM subtype) that acutely manifested as a unilateral sensory deficit following the first dose of an mRNA COVID-19 vaccine. LETM indicates a lesion that extends over three or more vertebral segments, as defined by a spinal MRI [2,7]. It is frequently associated with other autoimmune diseases, such as neuromyelitis optica (NMO) [7]. There were no anti-AQP4 antibodies in our patient, which ruled out the diagnosis of AQP4-Antibody-Positive NMO spectrum disease.

The most common neurological symptoms that occur after the COVID-19 vaccination are transient dizziness, headache, pain, muscle spasms, myalgia, and paresthesia [5]. Transverse myelitis after COVID-19 vaccination is rare (9 cases $/ 51,755,447$ dosages), according to ANA investigations [5]. A few cases of ATM were reported following vaccination with the recombinant ChAdOX1 nCoV-19 (AZD1222, Oxford/AstraZeneca, COVISHIELD ${ }^{\mathrm{TM}}$ ) vaccine [8-10]. As for mRNA COVID-19 vaccines, only one case has been reported online: a 63-year-old man who presented with distal cord myelitis following the second dose of the Moderna mRNA-1273 vaccine [11].

The temporal relationship between vaccination and ATM in our case was reasonable (48 h post-vaccination). A total of 119 cases of transverse myelitis that developed after vaccination were monitored during the period between 1985 and 2017 (29 men and 90 women; mean age: 32 years; age range: 12-61 years; mode 28 years) [12]. With regard to cases that occurred within the first six weeks after vaccination, the data suggest that the association between vaccination and some of these cases may not be coincidental [12]. The onset of neurological symptoms in our patient, at two days after COVID-19 vaccination, was shorter than that reported in previous case reports $[9,10]$. However, it was longer than that in the other reported case of myelitis related to the Moderna mRNA-1273 vaccine (onset of symptoms after $17 \mathrm{~h}$ ) [11].

Fever and pleocytosis after vaccination were observed in the present case. The clinical picture is consistent with the diagnosis of myelitis, with level 2 diagnostic certainty. According to the case definitions and guidelines for collection, analysis, and presentation of immunization safety data [6], for a level 1 diagnostic certainty of myelitis, acute cord inflammation should be demonstrated by histopathology. Level 2 diagnostic certainty requires the presence of symptomatic myelopathy in addition to at least two of the following indicators: fever up to $38^{\circ} \mathrm{C}$, a CSF pleocytosis of $>5 \mathrm{WBC} / \mathrm{mm}^{3}$, and neuroimaging findings indicating acute inflammation/demyelination of the spinal cord.

Post-vaccination myelitis has been reported in sporadic cases [13,14]. Infectious agents and vaccine adjuvants may evoke autoimmunity in similar ways, for instance, by molecular mimicry, epitope spreading, upregulation of cytokines, and polyclonal activation of B and T lymphocytes, which may induce immune reactions associated with ATM [13]. It is believed that the pathogenesis caused by adenoviral vaccines might be related to the use of chimpanzee adenovirus vectors [8,15]. However, the Moderna COVID-19 (mRNA1273) vaccine is composed of an mRNA vaccine encoding the pre-fusion spike protein encapsulated in lipid nanoparticles, with no adjuvants [16]. Therefore, other mechanisms might be involved in the development of autoimmunity in such cases. For instance, a study suggested that the immunological reaction between the SARS-CoV-2 spike protein antibody and tissue proteins, such as myelin basic protein, may be a plausible cause for the occurrence of demyelinating autoimmune diseases [17]. Furthermore, the inflammatory response triggered by the interaction between spike proteins and angiotensin-converting enzyme 2 (ACE2) receptors present in the endothelial cells of the blood-brain barrier or spinal neurons may be another possible mechanism of demyelination $[18,19]$.

Post-vaccination ATM-superimposed vitamin B12 deficiency was also observed in our patient. Nevertheless, the evidence suggested that the cervical lesions are more likely due to acute myelitis than subacute combined degeneration (SCD) [20]. In addition, the onset of sensory and gait disturbances was acute and not subacute. Furthermore, cervical MRI showed an eccentric (right-sided), ring-enhancing lesion, favoring a diagnosis of transverse myelitis, and not the typical inverted V sign of SCD [21,22]. Contrast enhancement of 
the spinal cord in our patient was also not typical of SCD [23]. Finally, the CSF showed pleocytosis, which suggested an inflammatory response and not SCD [20]. Since vitamin B12 is known as a cofactor of myelin formation as well as immunomodulation and neurotropism [24-26] while it could be a coincidental finding, the confounding role of vitamin B12 should not be underestimated.

Anti-myelin oligodendrocyte glycoprotein (MOG) antibodies were not examined in the patient due to our limitations. Because the MOG antibodies test is not including in Taiwan's national health insurance system, it cannot be afforded in testing for economic reasons. There were few cases with post-vaccination encephalomyelitis that were related to MOG antibodies [27,28]. As for our patient, there was no evidence of encephalitis. However, MOG antibody related disorders cannot be ruled out in our patient.

\section{Conclusions}

Here, we present a case of LETM, a scarcely reported disorder, that occurred shortly after receiving the first dose of an mRNA-based COVID-19 vaccine, with comorbid vitamin B12 deficiency. However, since LETM has rarely been associated with vaccination, the association of LETM with the Moderna COVID-19 (mRNA-1273) vaccine is still uncertain. There is a possibility that autoimmune reactions were triggered by the reaction between the SARS-CoV-2 spike protein antibody with tissue proteins, and the interaction between spike proteins and ACE2 receptors, as has been discussed earlier. However, the confounding role of vitamin B12 deficiency is unclear, and should be investigated in further reported cases and immunological studies.

Author Contributions: Conceptualization, J.-J.G. and C.-H.L.; Investigation, J.-J.G., H.-P.T., C.-L.L., J.-S.S., M.-H.L. and C.-H.L.; writing—original draft presentation, J.-J.G. and C.-H.L.; writing-review and editing, J.-J.G., H.-P.T., C.-L.L., J.-S.S., M.-H.L. and C.-H.L.; visualization, M.-H.L.; supervision, C.-H.L. All authors have read and agreed to the published version of the manuscript.

Funding: This research received no external funding.

Institutional Review Board Statement: Due to the case report nature of the manuscript, no ethical approval was required.

Informed Consent Statement: Written informed consent was obtained from the patient for the publication of this paper.

Data Availability Statement: The data presented in this study are available upon request from the corresponding author.

Conflicts of Interest: The authors declare no conflict of interest.

\section{References}

1. Kitley, J.L.; Leite, M.I.; George, J.S.; Palace, J.A. The differential diagnosis of longitudinally extensive transverse myelitis. Mult. Scler. J. 2012, 18, 271-285. [CrossRef]

2. Wingerchuk, D.M.; Weinshenker, B.G. Acute disseminated encephalomyelitis, transverse myelitis, and neuromyelitis optica. Continuum (Minneapolis, Minn.) 2013, 19, 944-967. [CrossRef]

3. Jeffery, D.R.; Mandler, R.N.; Davis, L.E. Transverse myelitis. Retrospective analysis of 33 cases, with differentiation of cases associated with multiple sclerosis and parainfectious events. Arch. Neurol. 1993, 50, 532-535. [CrossRef] [PubMed]

4. Baxter, R.; Lewis, E.; Goddard, K.; Fireman, B.; Bakshi, N.; DeStefano, F.; Gee, J.; Tseng, H.F.; Naleway, A.L.; Klein, N.P. Acute Demyelinating Events Following Vaccines: A Case-Centered Analysis. Clin. Infect. Dis. 2016, 63, 1456-1462. [CrossRef] [PubMed]

5. Goss, A.L.; Samudralwar, R.D.; Das, R.R.; Nath, A. ANA Investigates: Neurological Complications of COVID-19 Vaccines. Ann. Neurol. 2021, 89, 856-857. [CrossRef] [PubMed]

6. Sejvar, J.J.; Kohl, K.S.; Bilynsky, R.; Blumberg, D.; Cvetkovich, T.; Galama, J.; Gidudu, J.; Katikaneni, L.; Khuri-Bulos, N.; Oleske, J.; et al. Group. Encephalitis, myelitis, and acute disseminated encephalomyelitis (ADEM): Case definitions and guidelines for collection, analysis, and presentation of immunization safety data. Vaccine 2007, 25, 5771-5792. [CrossRef]

7. Weinshenker, B.G.; Wingerchuk, D.M.; Vukusic, S.; Linbo, L.; Pittock, S.J.; Lucchinetti, C.F.; Lennon, V.A. Neuromyelitis optica IgG predicts relapse after longitudinally extensive transverse myelitis. Ann. Neurol. 2006, 59, 566-569. [CrossRef] [PubMed] 
8. Román, G.C.; Gracia, F.; Torres, A.; Palacios, A.; Gracia, K.; Harris, D. Acute Transverse Myelitis (ATM): Clinical Review of 43 Patients With COVID-19-Associated ATM and 3 Post-Vaccination ATM Serious Adverse Events With the ChAdOx1 nCoV-19 Vaccine (AZD1222). Front. Immunol. 2021, 12, 653786. [CrossRef]

9. Malhotra, H.S.; Gupta, P.; Prabhu, V.; Garg, R.K.; Dandu, H.; Agarwal, V. COVID-19 vaccination-associated myelitis. QJM. 2021, hcab069. [CrossRef]

10. Pagenkopf, C.; Südmeyer, M. A case of longitudinally extensive transverse myelitis following vaccination against Covid-19. J. Neuroimmunol. 2021, 358, 577606. [CrossRef]

11. Fitzsimmons, W.; Nance, C.S. Sudden Onset of Myelitis after COVID-19 Vaccination: An Under-Recognized Severe Rare Adverse Event. 5 May 2021. Available online: http:/ / dx.doi.org/10.2139/ssrn.3841558 (accessed on 14 July 2021).

12. Shah, S.; Patel, J.M.; Alchaki, A.; Eddin, M.F.; Souayah, N. Development of Transverse Myelitis after Vaccination, A CDC/FDA Vaccine Adverse Event Reporting System (VAERS) Study, 1985-2017. (P5.099). Neurology 2018, 90 (Suppl. 15), P5.099.

13. Zanoni, G.; Nguyen, T.M.D.; Destefani, E.; Masala, L.; Nardelli, E.; Tridente, G. Transverse myelitis after vaccination. Eur. J. Neurol. 2002, 9, 696-697. [CrossRef] [PubMed]

14. Agmon-Levin, N.; Kivity, S.; Szyper-Kravitz, M.; Shoenfeld, Y. Transverse myelitis and vaccines: A multi-analysis. Lupus 2009, 18, 1198-1204. [CrossRef] [PubMed]

15. Austin, A.; Tincani, A.; Kivity, S.; Arango, M.-T.; Shoenfeld, Y. Transverse Myelitis Activation Post-H1N1 Immunization: A Case of Adjuvant Induction? Isr. Med. Assoc. J. 2015, 17, 120-122.

16. McDonald, I.; Murray, S.M.; Reynolds, C.J.; Altmann, D.M.; Boyton, R.J. Comparative systematic review and meta-analysis of reactogenicity, immunogenicity and efficacy of vaccines against SARS-CoV-2. NPJ Vaccines 2021, 6, 74. [CrossRef] [PubMed]

17. Vojdani, A.; Kharrazian, D. Potential antigenic cross-reactivity between SARS-CoV-2 and human tissue with a possible link to an increase in autoimmune diseases. Clin. Immunol. 2020, 217, 108480. [CrossRef] [PubMed]

18. Marino, S.; Taibi, R.; Pavone, P.; Marino, L.; Falsaperla, R. Neurotropism of SARS-CoV 2 and others coronavirus in children: Mechanisms and clinical manifestations. EJMO 2021, 5, 91-93. [CrossRef]

19. Nemoto, W.; Yamagata, R.; Nakagawasai, O.; Nakagawa, K.; Hung, W.Y.; Fujita, M.; Tadano, T.; Tan-No, K. Effect of spinal angiotensin-converting enzyme 2 activation on the formalin-induced nociceptive response in mice. Eur. J. Pharmacol. 2020, 5 , 172950. [CrossRef]

20. Goldish, D.; Massagli, T.L. Subacute Progressive Myelopathy: Transverse Myelitis or Subacute Combined Degeneration? A Case Report. PMER 2018, 10, 320-324. [CrossRef]

21. Matsuura, H.; Nakamura, T. Inverted V sign: Subacute combined degeneration of the spinal cord. QJM: Int. J. Med. 2018, 111, 65-66. [CrossRef]

22. Narra, R.; Mandapalli, A.; Jukuri, N.; Guddanti, P. "Inverted V sign" in Sub-Acute Combined Degeneration of Cord. J. Clin. Diagn. Res. 2015, 9, TJ01. [CrossRef] [PubMed]

23. Daroff, R. Structural Imaging Using Magnetic Resonance Imaging and Computed Tomography. In Bradley and Daroff's Neurology in Clinical Practice, 7th ed.; Joseph, J., John, C.M., Eds.; Elsevier: Amsterdam, The Netherlands, 2015; pp. 496-546.

24. Devi, A.; Singh, K.K.; Gupta, S.; Bhutani, N.; Agarwal, P. A descriptive study of clinical and radiological profile of longitudinal extensive myelitis in a tertiary hospital in Rajasthan, India. Clin. Neurol. Neurosurg. 2019, 181, 33-40. [CrossRef] [PubMed]

25. Krasnov, V.S.; Bedenko, A.S.; Totolyan, N.A.; Skoromets, A.A. Longitudinally extensive transverse myelitis associated with intrathecal synthesis of oligoclonal immunoglobulin G and with vitamin B12 deficiency. Neurol. Neuropsychiatry Psychosomatic. 2016, 8, 71-75. [CrossRef]

26. Miller, A.; Korem, M.; Almog, R.; Galboiz, Y. Vitamin B12, demyelination, remyelination and repair in multiple sclerosis. J Neurol. Sci. 2005, 233, 93-97. [CrossRef] [PubMed]

27. Kumar, N.; Graven, K.; Joseph, N.I.; Johnson, J.; Fulton, S.; Hostoffer, R.; Abboud, H. Case Report: Postvaccination Anti-Myelin Oligodendrocyte Glycoprotein Neuromyelitis Optica Spectrum Disorder: A Case Report and Literature Review of Postvaccination Demyelination. Int. J. MS Care 2020, 22, 85-90. [CrossRef]

28. Jarius, S.; in cooperation with the Neuromyelitis Optica Study Group (NEMOS); Ruprecht, K.; Kleiter, I.; Borisow, N.; Asgari, N.; Pitarokoili, K.; Pache, F.; Stich, O.; Beume, L.-A.; et al. MOG-IgG in NMO and related disorders: A multicenter study of 50 patients. Part 2: Epidemiology, clinical presentation, radiological and laboratory features, treatment responses, and long-term outcome. J. Neuroinflammation 2016, 13, 280. [CrossRef] [PubMed] 